\title{
Application of Artificial Intelligence in Chemistry
}

\author{
Preeti Rai $^{{ }^{*} \text {, Harsha Chatrath }}{ }^{2}$
}

${ }^{1 *}$ Research scholar, JJT University Jhunjhunu, Rajasthan, India; ${ }^{2}$ Research Guide JJT University Jhunjhunu, Rajasthan, India.

Email: ${ }^{1 *}$ rai.prit2010@gmail.com, ${ }^{2}$ harshamohini@gmail.com

DOI: https://doi.org/10.18510/gctl.2021.721

\section{Article History: Received on $02^{\text {nd }}$ November 2021, revised on $20^{\text {th }}$ December 2021, published on $23^{\text {rd }}$ December 2021}

All the problems can be solved with the help of machines mainly computers using algorithm and by interpreting their output data is considered as artificial intelligence (AI). Artificial intelligence is faster than manual work, reduces manpower, more efficient and accurate and used in various field these days and coming up with more advanced technology. With the help of artificial intelligence, drugs can be formulated and produced in an advanced way. New machineries' used in chemical or pharmaceutical labs are much advanced these days, that reduces the time of the analysis.

There is a strong bond between artificial intelligence and chemistry. In the field of chemistry designing new molecules, molecular property detection of molecules and compounds, drug discovery, synthesis and retrosynthesis of molecules, analysis prediction for better and accurate results, all these can be done with the help of artificial intelligence.

Artificial intelligence can help to separate and combine the molecules through pre-existing data base and giving novel and different results by omitting the least productive and undesirable results (Soumyaa Rawat, 2021, AI).

Artificial intelligence is not only limited to chemical labs in terms of its applications, but it is also useful for pharmaceuticals, medicines, advanced analytical techniques, healthcare industries, bio-chemistry and other related fields.

Molecular properties of new molecules can be detected, compared and predicted with pre-existing database, reduces the time for analysis and comparison and efficient results are produced by artificial intelligence.

The designing and discovery of new molecules or materials, to predict and decide the routes of reactions for synthesis and retrosynthesis of molecules, the modeling of processes and the support for sensorial analysis are the four domains which was emphasized particularly in Chemical Product Engineering (CPE).

Retrosynthesis of molecules consume enormous time and energy, which is reduced by artificial intelligence, gives accurate and efficient results in less time. AI algorithms help to predict probable and accurate analytical methods, to get fast and accurate result.

Deep learning (DL) helped to study and explore the spaces present in small organic compounds and in materials during the last decade.

Halicin is a drug identified by using a machine-learning algorithm by the researchers of Massachusetts Institute of Technology (MIT). Halicin is an antibiotic compound effective against various strains of bacteria and E.coli.

S100A9 was identified as an potential inhibitors for various kind of diseases including cancer and Alzheimer's by using machine learning algorithms.

Theory of (physical) chemistry and information from the computer related to chemistry combinedly considered as "Chemoinformatics".

The prediction of chemical, biological or physical properties of molecules, the design of organic syntheses, and the elucidation of the structure of molecules all can be done with the help of Chemoinformatics, which makes chemist job easier.

Chemoinformatics has various applications in the field of agrichemical research, analytical chemistry, drug discovery, food science, material science, organic chemistry, process control and regulatory science.

Edible \& non-edible, adulterated \& non-adulterated and quality of Olive oil can be identified with the deep learning model of AI that is trained by using around 700 samples of Olive oil harvested in year 2014-2015 and 2015-2016.

Safety of novel drugs, future development and antioxidant activity can be assessed by using machine learning techniques.

A deep learning-based neural network model was developed to classify active and inactive compounds in relation to their ability to inhibit SAM-dependent methyltransferases.

Preparation of resins with specific strength and viscosity consumes more time as there can be different variables such as diols and diacids attached to the polyester group, solvents used for the dilution etc. Conformational structures of the polymer also changes with its properties. These all can be done by using QSPR method with the help of AI.

"e- Sweet" is a platform developed by machine-learning method to find out the sweetness (artificial sweetness) and relative sweetness (RS) of food automatically. 
Implementation of artificial intelligence (AI) methods can help to give more accurate results in terms of ADMET (absorption, distribution, metabolism, excretion, and toxicity of molecules or chemical compounds) and quantitative structure activity relationship (QSAR) models.

Virtual screening will be helpful for deciding the routes for the drug development, designing of drugs and will cut down the expenses and time.

Scientists of Stanford University developed a new artificial intelligence (AI) programme "Atom2Vec" that created the periodic table withing few hours after analysing the properties and differences between the elements from online database. (Shifa Naseer, Ker Than, 2018).

AI plays a major role in spectrochemistry also. A large number of database is available these days that helps to compare and analyse new compound in a short period of time.

Quantum chemistry combined with artificial intelligence or machine learning is helping to explore the spaces around and withing chemical compounds as well as to study atomization energies of organic compounds based on their nuclear charges and atomic positions.

The Planetary Instrument for X-ray Lithochemistry (PIXL) was attached to Perseverance's robotic arm using a hexapod and is guided by AI to get the most accurate aim for its X-ray beam to determine rock textures and structures. NASA also equipped the rover with a SuperCam that was used by AI to identify, zap, and vaporize rock targets with a pulsed laser to study their chemical makeup. (NASA - Mars Perseverance Press Kit, February 18, 2021).

By using attention-based neural networks, mapping the space of chemicals and chemical reactions can be done. (Philippe Schwaller, Teodoro Laino, and Alain Vaucher, January 28, 2021, IBM Research Blog).

\section{REFERENCES}

1. Trafton, A. (2020). Artificial intelligence yields new antibiotic, A deep-learning model identifies a powerful new drug that can kill many species of antibiotic-resistant bacteria, MIT News Office.

2. Bai, F., Hong, D., Lu, Y., Liu, H., Xu, C., \& Yao, X. (2019). Prediction of the Antioxidant Response Elements' Response of Compound by Deep Learning. Frontiers in Chemistry, 7. https://doi.org/10.3389/fchem.2019.00 $\underline{385}$

3. Cancilla, J. C., Torrecilla, J. S., Proestos, C. V., \& Valderrama, J. O. (2020). Editorial: Artificial Intelligence in Chemistry. Frontiers in Chemistry, 8. https://doi.org/10.3389/fchem.2020.00275

4. Gasteiger, J. (2020). Chemistry in Times of Artificial Intelligence. ChemPhysChem, 21(20), 2233-2242. https://doi.org/10.1002/cphc.202000518

5. Li, F., Wan, X., Xing, J., Tan, X., Li, X., Wang, Y., ... Zheng, M. (2019). Deep Neural Network Classifier for Virtual Screening Inhibitors of (S)-Adenosyl-L-Methionine (SAM)-Dependent Methyltransferase Family. Frontiers in Chemistry, 7. https://doi.org/10.3389/fchem.2019.00324

6. Molina, J., Laroche, A., Richard, J.-V., Schuller, A.-S., \& Rolando, C. (2019). Neural Networks Are Promising Tools for the Prediction of the Viscosity of Unsaturated Polyester Resins. Frontiers in Chemistry, 7. https://doi.org/10.3389/fchem.2019.00375

7. Panteleev, J., Gao, H., \& Jia, L. (2018). Recent applications of machine learning in medicinal chemistry. Bioorganic \& Medicinal Chemistry Letters, 28(17), 2807-2815. https://doi.org/10.1016/j.bmcl.2018.06.046

8. Trinh C, Meimaroglou D, Hoppe S.(2021). Machine Learning in Chemical Product Engineering: The State of the Art and a Guide for Newcomers. Processes, 9(8), 1456. https://doi.org/10.3390/pr9081456

9. Vega-Márquez, B., Nepomuceno-Chamorro, I., Jurado-Campos, N., \& Rubio-Escudero, C. (2020). Deep Learning Techniques to Improve the Performance of Olive Oil Classification. Frontiers in Chemistry, 7. https://doi.org/10.3389/fchem.2019.00929

10. Zheng, S., Chang, W., Xu, W., Xu, Y., \& Lin, F. (2019). e-Sweet: A Machine-Learning Based Platform for the Prediction of Sweetener and Its Relative Sweetness. Frontiers in Chemistry, 7. https://doi.org/10.3389/fchem.2019.00035 